\title{
Application of zeta-function techniques to the Compactified Gross-Neveu Model
}

\author{
Jorge M. C. Malbouisson ${ }^{* \dagger}$ \\ Instituto de Física, Universidade Federal da Bahia, 40210-340, Salvador, BA, Brazil \\ E-mail: jmalboui@ufba.br
}

\section{Faqir C. Khanna}

Theoretical Physics Institute, University of Alberta, Edmonton, AB T6G 2J1, Canada

E-mail: khanna@phys.ualberta.ca

\section{Adolfo P. C. Malbouisson}

Centro Brasileiro de Pesquisas Físicas/MCT, 22290-180, Rio de Janeiro, RJ, Brazil

E-mail: adolfoecbpf.br

\section{Ademir E. Santana}

Instituto de Física, Universidade de Brasília, 70910-900, Brasília, DF, Brazil

E-mail: asantanadif.unb.br

We consider the $N$-component $D$-dimensional Euclidean massive Gross-Neveu model, confined in a $(D-1)$-dimensional cubic box (edge $L$ ), at finite temperature $(T)$. Using $\zeta$-function analytical regularization, we determine the large- $N$ effective coupling constant $(g)$ as a function of $L, T$ and the fixed coupling constant $(\lambda)$, for the cases $D=2,3,4$. In all cases, we find that $g$ tends to 0 when $L$ goes to 0 or $T$ goes to infinity, corresponding to an "asymptotic freedom" type of behavior. For finite $L$ and $T$, distinct behaviors appear depending on the value of $\lambda$. For small $\lambda$ only "asymptotic freedom" occurs. However, for $\lambda$ greater than a "critical" value $\left(\lambda_{c}\right)$, starting from small values of $L$ (and low enough temperatures), a divergence of $g$ appears as $L$ approaches a value $L_{c}(\lambda)$ which lies in a finite interval for $\lambda \geq \lambda_{c}$. Such behavior suggests that the system becomes spatially confined in a box of size $L_{c}(\lambda)$ if $\lambda$ is large enough. If the temperature is raised, the divergence disappears at a temperature $T_{d}(\lambda)$ which can be considered as a deconfining temperature. Taking the fermionic mass as the constituent quark mass, the confining length and the deconfining temperature obtained are comparable with the estimated values for hadrons.

Fifth International Conference on Mathematical Methods in Physics - IC2006

April 24-28 2006

Centro Brasilerio de Pesquisas Fisicas, Rio de Janeiro, Brazil

\footnotetext{
${ }^{*}$ Speaker.

${ }^{\dagger}$ Work supported by CNPq (Brazil) and NSERC (Canada).
} 


\section{Introduction}

The difficulty of handling analytically QCD has stimulated, over the last decades, the use of phenomenological approaches and the study of effective, simplified, theories to get clues of the behavior of hadronic systems. In the realm of effective field theories, renormalizability is not a definitive requirement for a theoretical model to have a physical meaning. The simplest effective model which may be conceived to describe quark interactions, is a direct four-fermion coupling where the gluon fields are integrated out and all color degrees of freedom are ignored, in a way similar to the Fermi treatment of the weak interaction; this corresponds to the Gross-Neveu (GN) model [1], considered in space-time dimension $D=4$. In fact, the Gross-Neveu model is not renormalizable (perturbatively) for dimensions greater than $D=2$ but, for $D=3$, the $N$-component massive Gross-Neveu model has been constructed in the the large- $N$ limit [2].

In this report we generalize previous work on the 3- $D$ Gross-Neveu model with one compactified spatial dimension, at zero [3] and finite [4] temperatures, to arbitrary dimension $D$, studying particularly the cases $D=2,3,4$ with all spatial dimensions compactified. We consider the GN massive model in $D$ dimensions at finite temperature with $d(\leq D)$ compactified coordinates, one of them being the imaginary time whose compactification length is the inverse temperature. The compactification of spatial dimensions, engendered through a generalization of the Matsubara procedure (antiperiodic boundary conditions), correspond to consider the system contained in a parallelepiped "box" with bag model boundary conditions on its faces [5, 6]. In other words, our system is defined inside a spatial region in thermal equilibrium at some temperature. We study the behavior of the system as a function of its size and of the temperature. The large-N GN model, in arbitrary dimension $D$, will be regularized along the lines of the previous papers, that is, by subtracting polar terms coming from Epstein-Hurwitz generalized zeta-functions. We show that the model treated in this way has the same structure for all values of $D$, which allows us to conjecture that it would have a sense, in particular for the space-time dimension $D=4$. This assumption is reinforced a posteriori by the fact that the numerical results for the confining spatial dimensions and the deconfining temperature are of the same order of magnitude of the corresponding values for $D=2$ and $D=3$, and moreover are in the right ballpark of the experimentally measured values.

Similar ideas have been applied in different physical situations: for spontaneous symmetry breaking in the compactified $\phi^{4}$ model [7, 8]; for second-order phase transitions in superconducting films, wires and grains [9]; for the Casimir effect for bosons [10] and for fermions in a box [11]. For the Gross-Neveu model, discussed in the present paper, we obtain simultaneously asymptotic freedom type of behavior and spatial confinement, for low enough temperatures. We also show that, as the temperature is increased, a deconfining transition occurs. We calculate the values of the confining lengths and the deconfining temperature and compare the results with the values obtained from experiments and lattice calculations.

\section{Compactified Gross-Neveu model}

We consider the Wick-ordered, massive, $N$-component Gross-Neveu model in a $D$-dimensional Euclidean space, described by the Lagrangian density

$$
\mathscr{L}=: \bar{\psi}(x)(i \nabla+m) \psi(x):+\frac{u}{2}(: \bar{\psi}(x) \psi(x):)^{2},
$$


where $m$ is the mass, $u$ is the coupling constant, $x$ is a point of $\mathbf{R}^{D}$ and the $\gamma$ 's are the Dirac matrices. The quantity $\psi(x)$ is a spin $\frac{1}{2}$ field having $N$ (flavor) components, $\psi^{a}(x), a=1,2, \ldots, N$, and summation over flavor and spin indices is understood. We shall take the large- $N$ limit $(N \rightarrow \infty)$, which permits considerable simplification. We use natural units, $\hbar=c=k_{B}=1$.

The objective is to determine of the renormalized large- $N$ (effective) coupling constant when $d$ $(\leq D)$ Euclidean coordinates, say $x_{1}, \ldots, x_{d}$, are compactified. The compactification is engendered via a generalized Matsubara prescription, which corresponds to consider the system with topology $S^{1_{1}} \times \cdots S^{1_{d}} \times R^{D-d}$. In other words, the coordinates $x_{i}$ are restricted to segments of length $L_{i}(i=$ $1,2, \ldots d)$, with the field $\psi(x)$ satisfying anti-periodic boundary conditions. If we choose one of the coordinates to represent the imaginary (Euclidean) time (say $x_{d}$ ), such scheme leads to the system at finite temperature, with $d-1$ compactified spatial dimensions; in this case, $L_{d}$ would stand for $\beta=1 / T$, the inverse of the temperature. Otherwise, with all $x_{i}$ referring to spatial coordinates, the model refers to compactified $d$ dimensions at $T=0$. For fermions, spatial compactification corresponds to the system be constrained by bag model boundary conditions (no outgoing currents) [5, 6], to "live" inside a $d$-dimensional parallelepiped "box" whose parallel faces are separated by distances $L_{i}(i=1,2, \ldots . d)[5,6]$. In any case, to describe the model with $d$ compactified Euclidean coordinates, the Feynman rules should be modified following the Matsubara replacements

$$
\int \frac{d k_{i}}{2 \pi} \rightarrow \frac{1}{L_{i}} \sum_{n_{i}=-\infty}^{+\infty}, \quad k_{i} \rightarrow \frac{2 \pi\left(n_{i}+\frac{1}{2}\right)}{L_{i}}, \quad i=1,2, \ldots, d .
$$

The large- $N$ effective coupling constant between the fermions will be defined in terms of the four-point function at zero external momenta. At leading order in $\frac{1}{N}$, summing chains of one-loop (bubble) diagrams, the $\left\{L_{i}\right\}$-dependent four-point function has the formal expression

$$
\Gamma_{D d}^{(4)}\left(0 ;\left\{L_{i}\right\}, u\right)=\frac{u}{1+N u \Sigma_{D d}\left(\left\{L_{i}\right\}\right)},
$$

where the $\left\{L_{i}\right\}$-dependent Feynman one-loop diagram is given by

$$
\Sigma_{D d}\left(\left\{L_{i}\right\}\right)=\frac{1}{L_{1} \cdots L_{d}} \sum_{\left\{n_{i}\right\}=-\infty}^{\infty} \int \frac{d^{D-d} k}{(2 \pi)^{D-d}}\left[\frac{m^{2}-\mathbf{k}^{2}-\sum_{i=1}^{d} v_{i}^{2}}{\left(\mathbf{k}^{2}+\sum_{i=1}^{d} v_{i}^{2}+m^{2}\right)^{2}}\right]
$$

in the above expression, $v_{i}=2 \pi\left(n_{i}+\frac{1}{2}\right) / L_{i}(i=1, \ldots, d)$ are the Matsubara frequencies and $\mathbf{k}$ stands for a continuous $(D-d)$-dimensional vector in momentum space.

To define a renormalized effective coupling constant, we have to handle the ultraviolet divergences of $\Sigma_{D d}\left(\left\{L_{i}\right\}\right)$. In order to simplify the use of regularization techniques, the following dimensionless quantities, $b_{i}=\left(m L_{i}\right)^{-2}(i=1, \ldots, d)$ and $q_{j}=k_{j} / 2 \pi m(j=d+1, \ldots, D)$, are introduced. In terms of these quantities, the one-loop diagram is written as

$$
\begin{aligned}
\Sigma_{D d}\left(\left\{b_{i}\right\}\right) & =\left.\Sigma_{D d}\left(s ;\left\{b_{i}\right\}\right)\right|_{s=2} \\
& =\left.\frac{m^{D-2}}{4 \pi^{2}} \sqrt{b_{1} \cdots b_{d}}\left\{\frac{1}{2 \pi^{2}} U_{D d}\left(s ;\left\{b_{i}\right\}\right)-U_{D d}\left(s-1 ;\left\{b_{i}\right\}\right)\right\}\right|_{s=2},
\end{aligned}
$$

where

$$
U_{D d}\left(\mu ;\left\{b_{i}\right\}\right)=\sum_{\left\{n_{i}\right\}=-\infty}^{\infty} \int \frac{d^{D-d} q}{\left[\mathbf{q}^{2}+\sum_{j=1}^{d} b_{j}\left(n_{j}+\frac{1}{2}\right)^{2}+(2 \pi)^{-2}\right]^{\mu}} .
$$


This shows explicitly that $\Sigma_{D d}$ has dimension of mass ${ }^{D-2}$, which is the inverse of the mass dimension of the coupling constant.

We shall use a modified minimal subtraction scheme, employing concurrently dimensional and analytical regularizations, where the terms to be subtracted are poles (for even $D \geq 2$ ) of the Epstein-Hurwitz zeta-functions [3]. Thus, performing the integral over $\mathbf{q}=\left(q_{d+1}, \ldots, q_{D}\right)$ in Eq. (2.6), using well-known dimensional regularization formulas, we obtain

$$
U_{D d}\left(\mu ;\left\{b_{i}\right\}\right)=\pi^{\frac{D-d}{2}} \frac{\Gamma\left(\mu-\frac{D-d}{2}\right)}{\Gamma(\mu)} \sum_{\left\{n_{i}\right\}=-\infty}^{\infty}\left[\sum_{j=1}^{d} b_{j}\left(n_{j}+\frac{1}{2}\right)^{2}+(2 \pi)^{-2}\right]^{\frac{D-d}{2}-\mu} .
$$

Transforming the summations over half-integers into sums over integers, Eq. (2.7) can be written as

$$
\begin{aligned}
U_{D d}\left(\mu ;\left\{b_{i}\right\}\right)= & \pi^{\frac{D-d}{2}} \frac{\Gamma\left(\mu-\frac{(D-d)}{2}\right)}{\Gamma(\mu)} 4^{\eta}\left[Z_{d}^{h^{2}}\left(\eta, b_{1}, \ldots, b_{d}\right)-\sum_{i=1}^{d} Z_{d}^{h^{2}}\left(\eta, \ldots, 4 b_{i}, \ldots\right)\right. \\
& \left.+\sum_{i<j=1}^{d} Z_{d}^{h^{2}}\left(\eta, \ldots, 4 b_{i}, \ldots, 4 b_{j}, \ldots\right)-\cdots+(-1)^{d} Z_{d}^{h^{2}}\left(\eta, 4 b_{1}, \ldots, 4 b_{d}\right)\right],(2.8
\end{aligned}
$$

where $h=\pi^{-1}, \eta=\mu-\frac{D-d}{2}$ and

$$
Z_{d}^{h^{2}}\left(\eta,\left\{a_{i}\right\}\right)=\sum_{\left\{n_{i}\right\}=-\infty}^{\infty}\left[\sum_{j=1}^{d} a_{j} n_{j}^{2}+h^{2}\right]^{-\eta}
$$

is the multiple ( $d$-dimensional) Epstein-Hurwitz zeta-function.

The function $Z_{d}^{h^{2}}\left(\eta,\left\{a_{i}\right\}\right)$ can be analytically extended to the whole complex $\eta$-plane [8], through a generalization of the procedure presented in Refs. [12, 13]; for reviews of applications of zeta-function regularization, see also Ref. [14]. We find (see Appendix)

$$
\begin{aligned}
Z_{d}^{h^{2}}\left(v,\left\{a_{i}\right\}\right)= & \frac{\pi^{d / 2}}{\sqrt{a_{1} \cdots a_{d}} \Gamma(v)}\left[\frac{1}{h^{2(v-d)}} \Gamma\left(v-\frac{d}{2}\right)\right. \\
& +4 \sum_{i=1}^{d} \sum_{n_{i}=1}^{\infty}\left(\frac{\pi n_{i}}{h \sqrt{a_{i}}}\right)^{v-\frac{d}{2}} K_{v-\frac{d}{2}}\left(\frac{2 \pi h n_{i}}{\sqrt{a_{i}}}\right) \\
& +8 \sum_{i<j=1}^{d} \sum_{n_{i}, n_{j}=1}^{\infty}\left(\frac{\pi}{h} \sqrt{\frac{n_{1}^{2}}{a_{1}}+\frac{n_{2}^{2}}{a_{2}}}\right)^{v-\frac{d}{2}} K_{v-\frac{d}{2}}\left(2 \pi h \sqrt{\frac{n_{1}^{2}}{a_{1}}+\frac{n_{2}^{2}}{a_{2}}}\right)+\cdots \\
& \left.+2^{d+1} \sum_{\left\{n_{i}\right\}=1}^{\infty}\left(\frac{\pi}{h} \sqrt{\frac{n_{1}^{2}}{a_{1}}+\cdots+\frac{n_{d}^{2}}{a_{d}}}\right)^{v-\frac{d}{2}} K_{v-\frac{d}{2}}\left(2 \pi h \sqrt{\frac{n_{1}^{2}}{a_{1}}+\cdots+\frac{n_{d}^{2}}{a_{d}}}\right)\right],
\end{aligned}
$$

where $K_{\alpha}(z)$ is the Bessel function of the third kind. This implies that the function $U_{D d}\left(\mu ;\left\{b_{i}\right\}\right)$ can also be analytically extended to the complex $\mu$-plane. Using the identity

$$
\sum_{j=1}^{N}\left(\frac{-1}{2}\right)^{j} \frac{N !}{j !(N-j) !}=\frac{1}{2^{N}}
$$


and grouping similar terms appearing in the parcels of Eq. (2.8), we find

$$
U_{D d}\left(\mu ;\left\{b_{i}\right\}\right)=\frac{2^{2 \mu-D} \pi^{2 \mu-\frac{D}{2}}}{\Gamma(\mu)} \frac{1}{\sqrt{b_{1} \cdots b_{d}}}\left[\Gamma\left(\mu-\frac{D}{2}\right)+2^{\frac{D}{2}} W_{D d}\left(\mu ;\left\{b_{i}\right\}\right)\right],
$$

with $W_{D d}\left(\mu ;\left\{b_{i}\right\}\right)$ given by

$$
W_{D d}\left(\mu ;\left\{b_{i}\right\}\right)=2^{1-\mu} \sum_{j=1}^{d} 2^{2 j} \sum_{\left\{\rho_{j}\right\}} \sum_{\left\{c_{\rho_{k}}=1,4\right\}}\left(\prod_{k=1}^{j} \frac{(-1)^{c_{\rho_{k}}}-1}{\sqrt{c_{\rho_{k}}}}\right) F_{D j}\left(\mu ; c_{\rho_{1}} b_{\rho_{1}}, \ldots, c_{\rho_{j}} b_{\rho_{j}}\right),
$$

where $\left\{\rho_{j}\right\}$ stands for the set of all combinations of the indices $\{1,2, \ldots, d\}$ with $j$ elements and the functions $F_{D j}\left(\mu ; a_{1}, \ldots, a_{j}\right)(j=1, \ldots, d)$ are defined by

$$
F_{D j}\left(\mu ; a_{1}, \ldots, a_{j}\right)=\sum_{n_{1}, \ldots, n_{j}=1}^{\infty}\left(2 \sqrt{\frac{n_{1}^{2}}{a_{1}}+\cdots+\frac{n_{j}^{2}}{a_{j}}}\right)^{\mu-\frac{D}{2}} K_{\mu-\frac{D}{2}}\left(2 \sqrt{\frac{n_{1}^{2}}{a_{1}}+\cdots+\frac{n_{j}^{2}}{a_{j}}}\right) .
$$

The use of Eq. (2.11) leads directly to an analytic extension of $\Sigma_{D d}\left(s ;\left\{b_{i}\right\}\right)$ for complex values of $s$, within a vicinity of $s=2$ :

$$
\begin{aligned}
\Sigma_{D d}\left(s ;\left\{b_{i}\right\}\right)= & \frac{m^{D-2} \pi^{\frac{D}{2}}}{(2 \pi)^{D-2(s-2)} \Gamma(s)}\left\{(s-1-D) \Gamma\left(s-1-\frac{D}{2}\right)\right. \\
& \left.+\left[2^{\frac{D}{2}+1} W_{D d}\left(s ;\left\{b_{i}\right\}\right)-(s-1) 2^{\frac{D}{2}} W_{D d}\left(s-1 ;\left\{b_{i}\right\}\right)\right]\right\} .
\end{aligned}
$$

We notice that the first term in this expression for $\Sigma_{D d}\left(s ;\left\{b_{i}\right\}\right)$, involving the $\Gamma$-functions, does not depend on parameters $b_{i}$, that is, it is independent of the compactification lengths $L_{i}(i=1, \ldots, d)$. For even dimensions $D \geq 2$, this term is divergent due to the poles of the $\Gamma$-functions. Since we are using a modified minimal subtraction scheme, where terms to be subtracted are poles appearing at the physical value $s=2$, this term should be suppressed to give the renormalized single bubble function, $\Sigma_{D d}^{R}\left(\left\{b_{i}\right\}\right)$. For the sake of uniformity, this term is also subtracted in the case of odd dimensions, where no poles of the $\Gamma$-functions are present; in a such situation, we perform a finite renormalization. The second term, which depends on the compactification lengths $L_{i}$ and arises from the regular part of the analytical extension of the Epstein-Hurwitz zeta-functions, gives the renormalized one-loop diagram

$$
\Sigma_{D d}^{R}\left(\left\{b_{i}\right\}\right)=\frac{m^{D-2}}{(2 \pi)^{\frac{D}{2}}}\left[2 W_{D d}\left(2 ;\left\{b_{i}\right\}\right)-W_{D d}\left(1 ;\left\{b_{i}\right\}\right)\right] .
$$

Notice that, replacing $b_{i}$ by $\left(m L_{i}\right)^{-2}$ in the above expression, we recover explicitly $\Sigma_{D d}^{R}\left(\left\{L_{i}\right\}\right)$. Now, we proceed to analyze the behavior of the large- $N$ coupling constant in various cases.

\section{Large- $N$ renormalized coupling constant}

As it is usual in four-body interacting field theories, we shall define the coupling constant in terms of the four-point function at fixed external momenta, taking $\mathbf{p}=0$. The coupling constant is

then interpreted as measuring the strength of the interaction between the fermions. Thus, inserting 
$\Sigma_{D d}^{R}\left(\left\{b_{i}\right\}\right)$ into Eq. (2.3) and taking the limit $N \rightarrow \infty$ and $u \rightarrow 0$, with $N u=\lambda$ fixed as usual, we find the large- $N$ ( $\left\{b_{i}\right\}$-dependent) renormalized coupling constant, for $d(\leq D)$ compactified dimensions, as

$$
g_{D d}\left(\left\{b_{i}\right\}, \lambda\right)=\lim _{N \rightarrow \infty}\left[N \Gamma_{D d}^{(4) R}\left(0,\left\{b_{i}\right\}, u\right)\right]=\frac{\lambda}{1+\lambda \Sigma_{D d}^{R}\left(\left\{b_{i}\right\}\right)} .
$$

This is the basic result for subsequent analysis. Some general properties of the renormalized effective coupling constant can be obtained from the fact that the dependence of $\Sigma_{D d}^{R}$ on $\left\{b_{i}\right\}$ is dictated by the Bessel functions of the third kind appearing in Eq. (2.13).

First, notice that if all $b_{i}$ tend to zero, that is, if $\left\{L_{i} \rightarrow \infty\right\}$, then $\Sigma_{D d}^{R} \rightarrow 0$ and therefore

$$
\lim _{\left\{L_{i} \rightarrow \infty\right\}} g_{D d}\left(\left\{b_{i}\right\}, \lambda\right)=\lambda
$$

This is an expected result, expressing a consistency condition for our calculations: when all the compactification lengths tend to infinity, the renormalized effective coupling constant must reduce to the renormalized fixed coupling constant in free space at zero temperature, $\lambda$. On the other hand, if any of the $b_{i}$ tends to $\infty$ (that is, if any compactification length $L_{i}$ goes to 0 ), the renormalized single bubble diagram $\Sigma_{D d}^{R}$ diverges, implying in the vanishing of the renormalized effective coupling constant $g_{D d}$, irrespective of the value of $\lambda$. This suggests that the system presents an ultraviolet asymptotic-freedom type of behavior for short distances and/or for high temperatures.

Interesting features should appear if $\Sigma_{D d}^{R}$ acquires negative values; in such a situation, depending on the value of $\lambda$, the renormalized effective coupling constant may diverge for finite values of the lengths $L_{i}$. Such a possibility, and its consequences, will be explicitly investigated in the following subsections, where we consider, particularly, the compactified model for space-time dimensions $D=2,3,4$ at zero temperature. The discussion of finite temperature effects is postponed to Sec. III.

\subsection{Compactified Gross-Neveu model in $D=2$ at $T=0$}

Firstly, consider the case of a two-dimensional space-time $(D=2)$, where only the spatial coordinate is compactified, that is $d=1$, and fix $L_{1}=L$. Inserting these values of $D$ and $d$ into Eqs. (2.12) and (2.13), remembering that $b_{1}=b=(m L)^{-2}$, Eq. (2.15) becomes

$$
\Sigma_{21}^{R}(L)=2 E_{1}(2 m L)-E_{1}(m L)
$$

where the function $E_{1}(x)$ is given by

$$
E_{1}(x)=\frac{1}{\pi} \sum_{n=1}^{\infty}\left[(x n) K_{1}(x n)-K_{0}(x n)\right]
$$

As stated before, $\Sigma$ and $\lambda$ are dimensionless for $D=2$. Notice that $L$ has dimension of inverse of mass and so the argument of the Bessel functions are dimensionless, as it should. We can calculate $\Sigma_{21}^{R}(L)$ numerically by truncating the series in Eq. (3.4), defining the function $E_{1}(y)$, at some value $n=N$. For moderate and large values of $m L$ (say, $\gtrsim 0.5$ ), $N$ can be taken as a relatively small value; for example, for $m L=0.5$ with $N=36$, we obtain the correct value of $\Sigma_{21}^{R}$ to six decimal places. 


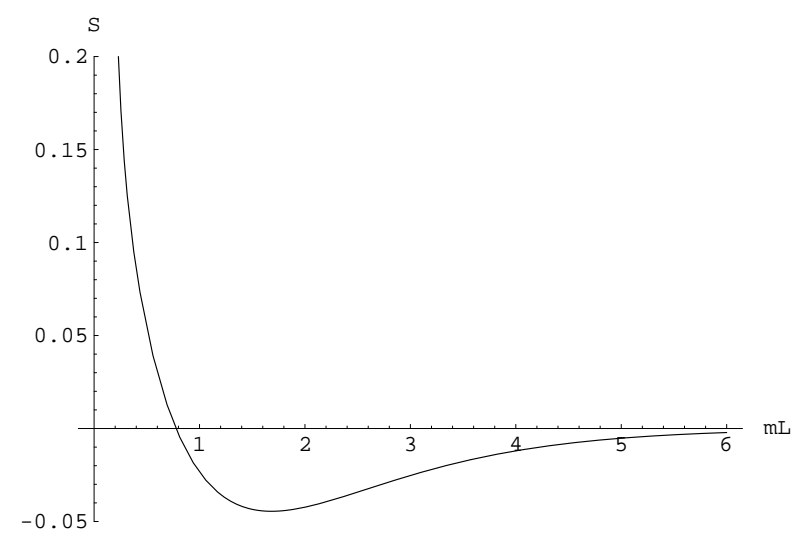

Figure 1: Plot of $S=\Sigma_{21}^{R}(L)$ as a function of $m L$.

However, due to the presence of positive and negative parcels in the summation and the fact that the functions $K_{0}(z)$ and $K_{1}(z)$ diverge for $z \rightarrow 0$, large values of $N$ are required to calculate $\Sigma_{21}^{R}$ for small values of $m L$; for $m L=0.005$, we have to take $N=4500$ to obtain $\Sigma_{21}^{R}$ to six decimal places.

Some features about the function $\Sigma_{21}^{R}(L)$ can be obtained from the numerical treatment of Eq. (3.3) and can also be visualized from from Fig. 1, where this quantity is plotted as a function of $m L$. As already remarked on general grounds, $\Sigma_{21}^{R}(L)$ diverges $(\rightarrow+\infty)$ when $L \rightarrow 0$ and tends to 0 , through negative values, as $L \rightarrow \infty$. We find that $\Sigma_{21}^{R}(L)$ vanishes for a specific value of $L$, which we denote by $L_{\mathrm{min}}^{(2)}$, being negative for all $L>L_{\mathrm{min}}^{(2)}$; it also assumes a minimum (negative) value for a value of $L$ we denote by $L_{\max }^{(2)}$, for reasons that will be clarified later. Numerically, we find: $L_{\min }^{(2)} \simeq 0.78 m^{-1} ; L_{\max }^{(2)} \simeq 1.68 m^{-1}$ and $\Sigma_{21}^{R \min } \simeq-0.0445$. This dependence of $\Sigma_{21}^{R}$ on $L$, in particular the fact that $\Sigma_{21}^{R}(L)$ is negative for $L>L_{\mathrm{min}}^{(2)}$, has remarkable influence on the behavior of the renormalized effective coupling constant.

In the present case, Eq. (3.1) becomes

$$
g_{21}(L, \lambda)=\frac{\lambda}{1+\lambda \Sigma_{21}^{R}(L)}
$$

We first note that, independent of the value of $\lambda, g_{21}(L, \lambda)$ approaches 0 as $L \rightarrow 0$; therefore, the system presents a kind of asymptotic-freedom behavior for short distances. On the other hand, starting from a low value of $L$ (within the region of asymptotic freedom) and increasing the size of the system, $g_{21}$ will present a divergence at a finite value of $L\left(L_{c}^{(2)}\right)$, if the value of the fixed coupling constant $(\lambda)$ is high enough. In fact, this will happen for all values of $\lambda$ above the "critical value" $\lambda_{c}^{(2)}=\left(-\Sigma_{21}^{R \min }\right)^{-1} \simeq 22.5$. We interpret this result by stating that, in the strong-coupling regime $\left(\lambda>\lambda_{c}^{(2)}\right)$ the system gets spatially confined in a segment of length $L_{c}^{(2)}(\lambda)$. The behavior of the effective coupling as a function of $m L$ is illustrated in Fig. 2, for some values of the fixed coupling constant $\lambda$.

From the definition of $\lambda_{c}^{(2)}$, we find that, for $\lambda=\lambda_{c}^{(2)}$, the divergence of $g_{21}(L, \lambda)$ is reached as $L$ approaches the value that makes $\Sigma_{21}^{R}$ minimal, which we denoted by $L_{\max }^{(2)}$. On the other hand, since $g_{21}(L, \lambda \rightarrow \infty)=\Sigma_{21}^{R}(L), L_{c}^{(2)}(\lambda)$ tends to $L_{\min }^{(2)}$, the zero of $\Sigma_{21}^{R}$, as $\lambda \rightarrow \infty$. In other 


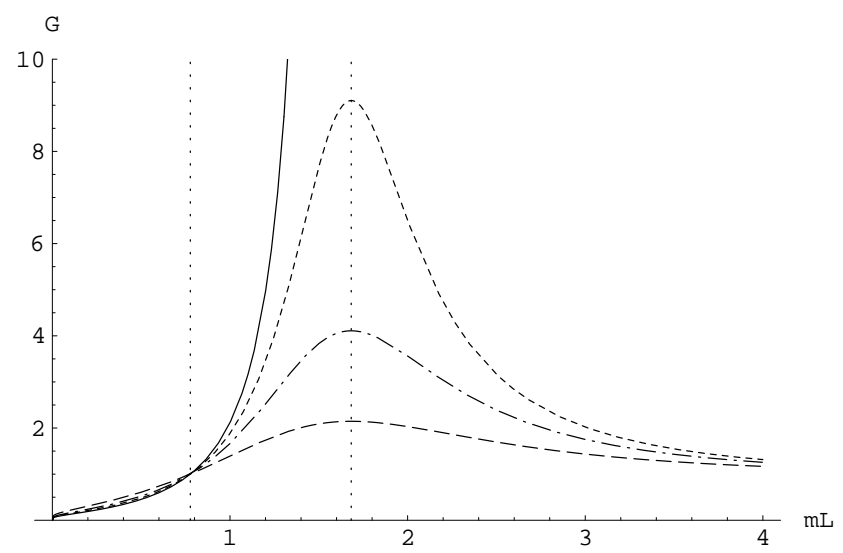

Figure 2: Plots of the relative effective coupling constant, $G=g_{21}(L, \lambda) / \lambda$, as a function of $m L$ for some values of $\lambda: 12.0$ (dashed line), 17.0 (dotted-dashed line), 20.0 (dotted line) and 22.5 (full line). The dotted vertical lines, passing by $L_{\min }^{(2)} \simeq 0.78 \mathrm{~m}^{-1}$ and $L_{\max }^{(2)} \simeq 1.68 \mathrm{~m}^{-1}$, are plotted as a visual guide.

words, the confining length $L_{c}^{(2)}(\lambda)$ pertains to the interval $\left(L_{\min }^{(2)}, L_{\max }^{(2)}\right]$. For a given value of $\lambda$, the confining length $L_{c}^{(2)}(\lambda)$ can be found numerically by determining the smallest solution of the equation $1+\lambda \Sigma_{21}^{R}(L)=0$. These results are presented in Fig. 3, where we plot $m L_{c}^{(2)}(\lambda)$ as a function of $l=\lambda / \lambda_{c}^{(2)}$.

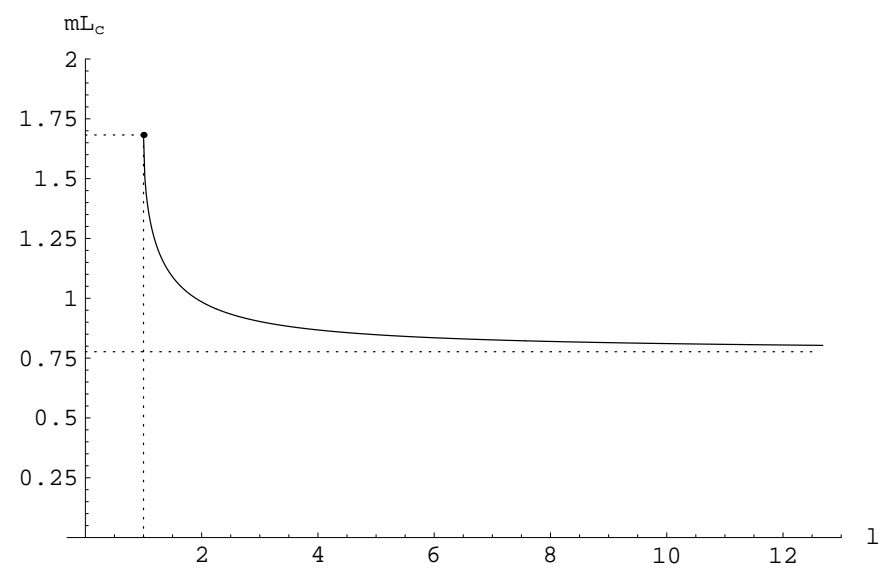

Figure 3: Plot of the confining length (in units of $m^{-1}$ ), as a function of $l=\lambda / \lambda_{c}^{(2)}$; the horizontal dashed lines correspond to the limiting values $m L_{\min }^{(2)} \simeq 0.78$ and $m L_{\max }^{(2)} \simeq 1.68$.

\subsection{Compactified 3- $D$ Gross-Neveu model at $T=0$}

We start by considering the 3-D model at zero temperature, with two compactified dimensions. We should then take $D=3$ and $d=2$ in formulas (2.12-2.15), with $L_{1}$ and $L_{2}$ being the compactification lengths associated with the two spatial coordinates $x_{1}$ and $x_{2}$, measured in units of $m^{-1}\left(\sqrt{b_{1}}\right.$ and $\sqrt{b_{2}}$, respectively). Using that $K_{ \pm \frac{1}{2}}(z)=\sqrt{\pi} \exp (-z) / \sqrt{2 z}$ and summing geometric series, we 
find the following expression for the renormalized bubble diagram

$$
\begin{aligned}
\frac{\sum_{32}^{R}\left(L_{1}, L_{2}\right)}{m}= & \frac{1}{2 \pi}\left[\frac{1}{L_{1}} \log \left(1+e^{-L_{1}}\right)+\frac{1}{L_{2}} \log \left(1+e^{-L_{2}}\right)\right] \\
& -\frac{1}{2 \pi}\left[\frac{1}{1+e^{L_{1}}}+\frac{1}{1+e^{L_{2}}}\right] \\
& +\frac{1}{\pi}\left[G_{2}\left(L_{1}, L_{2}\right)-2 G_{2}\left(L_{1}, 2 L_{2}\right)-2 G_{2}\left(2 L_{1}, L_{2}\right)+4 G_{2}\left(2 L_{1}, 2 L_{2}\right)\right],
\end{aligned}
$$

where the function $G_{2}(x, y)$ is defined by

$$
G_{2}(x, y)=\sum_{n, l=1}^{\infty} \exp \left(-\sqrt{x^{2} n^{2}+y^{2} l^{2}}\right)\left[1-\frac{1}{\sqrt{x^{2} n^{2}+y^{2} l^{2}}}\right] .
$$

Notice that the numerical computation of $\Sigma_{32}^{R}\left(L_{1}, L_{2}\right)$ is greatly facilitated by the fact that the double series defining the function $G_{2}(y, z)$ is rapidly convergent.

We remark, initially, that by taking one of the compactifications lengths going to infinity in the expression (3.6), all terms depending on it vanish and we regain the renormalized bubble diagram for the case where only one spatial dimension is compactified in the 3-D model; thus, particularly, all the results of Ref. [3] follow. We see explicitly that if both $L_{1}$ and $L_{2}$ tend simultaneously to $\infty, \Sigma_{32}^{R}$ goes to zero and $g_{32} \rightarrow \lambda$, confirming the general statement made above. Also, if either $L_{1}$ or $L_{2}$ tends to $0, \Sigma_{32}^{R} \rightarrow+\infty$ implying that the system gets asymptotically free, with the effective coupling constant vanishing in this limit. However, instead of work in more general grounds, we shall restrict our analysis to the case where the system is confined within a square of size $L$, by considering $L_{1}=L_{2}=L$ and without loosing the generality our results may have.

The quantity $\Sigma_{32}^{R}(L, L) / m$ behaves, as a function of $L$ (measured in units of $m^{-1}$ ), in the same way as it appears in Fig 1. We find that it vanishes for a specific value of $L, L_{\mathrm{min}}^{(3)}$, being negative for all $L \geq L_{\mathrm{min}}^{(3)}$; it also assumes an absolute minimum (negative) value for a value of $L$ denoted by $L_{\max }^{(3)}$. Numerically, we find: $L_{\min }^{(3)} \simeq 1.30 m^{-1}, L_{\max }^{(3)} \simeq 2.10 m^{-1}$ and $\Sigma_{32}^{R \min } \simeq-0.0099 m$. This behavior of $\Sigma_{32}^{R}(L, L)$ has deep implications on the renormalized effective coupling constant.

In fact, in the present case, Eq. (3.1) reduces to $g_{32}(L, \lambda)=\lambda /\left[1+\lambda \Sigma_{32}^{R}(L)\right]$ and we find that, for $\lambda \geq \lambda_{c}^{(3)}=\left(-\Sigma_{32}^{R \min }\right)^{-1} \simeq 101 \mathrm{~m}^{-1}$, the denominator vanishes for a finite value of $L$, $L_{c}^{(3)}(\lambda)$, leading to a divergence in the renormalized effective coupling constant. The behavior of the effective coupling as a function of $L$, for increasing values of the fixed coupling constant $\lambda$, can be illustrated showing the same pattern as that of Fig. 2 for the preceding case. We find that the divergence occurs at $L_{c}^{(3)}(\lambda) \in\left(L_{\min }^{(3)}, L_{\max }^{(3)}\right]$. Again, we interpret such a result by considering the system spatially confined in the sense that, starting with $L$ small (in the region of asymptotic freedom), the size of the square can not go above $L_{c}^{(3)}(\lambda)$ since $g_{32}(L, \lambda) \rightarrow \infty$ as $L \rightarrow L_{c}^{(3)}(\lambda)$. The confining length $L_{c}^{(3)}(\lambda)$ is given by the smallest root of the equation $1+\lambda \Sigma_{32}^{R}(L)=0$; its behavior as a function of the relative effective coupling constant, $l=\lambda / \lambda_{c}^{(3)}$, follows the same trend as in that in Fig. 3 but with the limiting values $L_{\min }^{(3)} \simeq 1.30 m^{-1}$ and $L_{\max }^{(3)} \simeq 2.10 m^{-1}$.

\section{3 $D=4$ case at zero temperature}

Let us now consider the 4-dimensional Gross-Neveu model where all three spatial coordinates have been compactified. Replacing all $b_{i}^{-1}$ by $L^{-2}$ (again, measured in units of $m^{-1}$ ) in Eqs. (2.12. 
2.15), which corresponds to consider the system confined within a cubic box, we obtain

$$
\begin{aligned}
\Sigma_{43}^{R}(L)= & m^{2}\left[6 H_{1}(2 L)-3 H_{1}(L)+6 H_{2}(L, L)\right. \\
& -24 H_{2}(L, 2 L)+24 H_{2}(2 L, 2 L)-4 H_{3}(L, L, L) \\
& \left.+24 H_{3}(L, L, 2 L)-48 H_{3}(L, 2 L, 2 L)+32 H_{3}(2 L, 2 L, 2 L)\right] .
\end{aligned}
$$

where the functions $H_{j}, j=1,2,3$, are defined by

$$
\begin{aligned}
H_{1}(x) & =\frac{1}{2 \pi^{2}} \sum_{n=1}^{\infty}\left[K_{0}(x n)-\frac{K_{1}(x n)}{(x n)}\right], \\
H_{2}(x, y) & =\frac{1}{2 \pi^{2}} \sum_{n, l=1}^{\infty}\left[K_{0}\left(\sqrt{x^{2} n^{2}+y^{2} l^{2}}\right)-\frac{K_{1}\left(\sqrt{x^{2} n^{2}+y^{2} l^{2}}\right)}{\left(\sqrt{x^{2} n^{2}+y^{2} l^{2}}\right)}\right] \\
H_{3}(x, y, z) & =\frac{1}{2 \pi^{2}} \sum_{n, l, r=1}^{\infty}\left[K_{0}\left(\sqrt{x^{2} n^{2}+y^{2} l^{2}+z^{2} r^{2}}\right)-\frac{K_{1}\left(\sqrt{x^{2} n^{2}+y^{2} l^{2}}\right)}{\left(\sqrt{x^{2} n^{2}+y^{2} l^{2}+z^{2} r^{2}}\right)}\right] .
\end{aligned}
$$

The quantity $\Sigma_{43}^{R}(L) / m^{2}$ has the same behavior as its counterparts for $D=2$ and $D=3$. We find numerically that $\Sigma_{43}^{R}(L)$ vanishes for $L=L_{\min }^{(4)} \simeq 1.68 \mathrm{~m}^{-1}$, being negative for $L>L_{\min }^{(4)}$, and assumes the minimum value, $\Sigma_{43}^{R \min } \simeq-0.002275 \mathrm{~m}^{2}$, when $L=L_{\max }^{(4)} \simeq 2.37 \mathrm{~m}^{-1}$.

As in the other cases discussed in detail before, the renormalized effective coupling constant, $g_{43}(L, \lambda)=\lambda /\left[1+\lambda \Sigma_{43}^{R}(L)\right]$, diverges at a finite value of $L, L_{c}^{(4)}(\lambda)$, if $\lambda \geq \lambda_{c}^{(4)}=-\left(\Sigma_{43}^{R \min }\right)^{-1} \simeq$ $439.5 \mathrm{~m}^{-2}$, meaning that the system gets confined in a cubic box of edge $L_{c}^{(4)}(\lambda) \in\left(L_{\min }^{(4)}, L_{\max }^{(4)}\right]$. The plot of $L_{c}^{(4)}(\lambda)$, as a function of $l=\lambda / \lambda_{c}^{(4)}$, shows the similar features as that of Fig. 3, but with the limiting values $L_{\min }^{(4)} \simeq 1.68 \mathrm{~m}^{-1}$ and $L_{\max }^{(4)} \simeq 2.37 \mathrm{~m}^{-1}$.

\section{Effect of temperature on the compactified Gross-Neveu model}

We now discuss the effect of raising the temperature on the renormalized effective coupling constant for the Gross-Neveu model with all spatial dimensions compactified. Finite temperature is introduced through the compactification of the time coordinate, with the compactification length given by $L_{D}=\beta=1 / T$. Although in an Euclidean theory time and space coordinates are treated on the same footing, the interpretation of their compactifications are rather distinct. On general grounds, we expect that the dependence of $\Sigma_{D}^{R}$ and $g_{D}$ on $\beta$ should follow similar patterns as that for the dependence with $L$. In fact, as $\beta \rightarrow 0$ (that is, $T \rightarrow \infty$ ), $\Sigma_{D}^{R} \rightarrow \infty$ implying that $g_{D} \rightarrow 0$, independently of the value of the fixed coupling constant $\lambda$; thus, we have asymptotic-freedom behavior for very high temperatures. Therefore, we expect that, starting from the compactified model at $T=0$ with $\lambda \geq \lambda_{c}^{(D)}$, raising the temperature will lead to the suppression of the divergence of $g_{D}$ and the consequent deconfinement of the system. In this section, we discuss this deconfining transition and determine the deconfining temperature for the cases of $D=2,3,4$.

\section{1 $D=2$}

We now consider the effect of finite temperature on the 2-D compactified model. For that, we take the second Euclidean coordinate (the imaginary time, $x_{2}$ ) compactified in a length $L_{2}=\beta=$ 
$1 / T, T$ being the temperature. In this case, the $L$ and $\beta$-dependent bubble diagram, obtained from Eqs. (2.13 2.15) with $b_{1}=L^{-2}$ and $b_{2}=\beta^{-2}\left(L\right.$ and $\beta$ measured in units of $\left.m^{-1}\right)$, can be written as

$$
\begin{aligned}
\Sigma_{22}^{R}(L, \beta)= & 2 E_{1}(2 L)-E_{1}(L)+2 E_{1}(2 \beta)-E_{1}(\beta) \\
& +2 E_{2}(L, \beta)-4 E_{2}(2 L, \beta)-4 E_{2}(L, 2 \beta)+8 E_{2}(2 L, 2 \beta),
\end{aligned}
$$

where the function $E_{1}(x)$ is given by Eq. (3.4) and the function $E_{2}(x, y)$ is defined by

$$
E_{2}(x, y)=\frac{1}{\pi} \sum_{n, l=1}^{\infty}\left\{\left(\sqrt{x^{2} n^{2}+y^{2} l^{2}}\right) K_{1}\left(\sqrt{x^{2} n^{2}+y^{2} l^{2}}\right)-K_{0}\left(\sqrt{x^{2} n^{2}+y^{2} l^{2}}\right)\right\} .
$$

Firstly, observe that if $\beta \rightarrow \infty$, all terms depending on $\beta$ vanishes and $\Sigma_{22}^{R}(L, \beta)$ reduces to the expression for zero temperature, $\Sigma_{21}^{R}(L)$. On the other hand, independently of the value of $\lambda$, if $\beta \rightarrow 0, \Sigma_{22}^{R}(L, \beta) \rightarrow \infty$ and the system becomes asymptotically free; therefore, we expect that raising the temperature tends to suppress the divergence of $g$, favoring the disappearance of the mentioned spatial confinement. Such a reasoning implies that, for a given value of $\lambda \geq \lambda_{c}^{(2)}$, there exists a temperature, $T_{d}^{(2)}(\lambda)$, at which the divergence in $g$ disappears and the system becomes spatially unconfined. We can determine $T_{d}^{(2)}(\lambda)$ by analyzing the behavior of $g_{22}^{-1}(L, \beta, \lambda)$ as $T$ is increased.

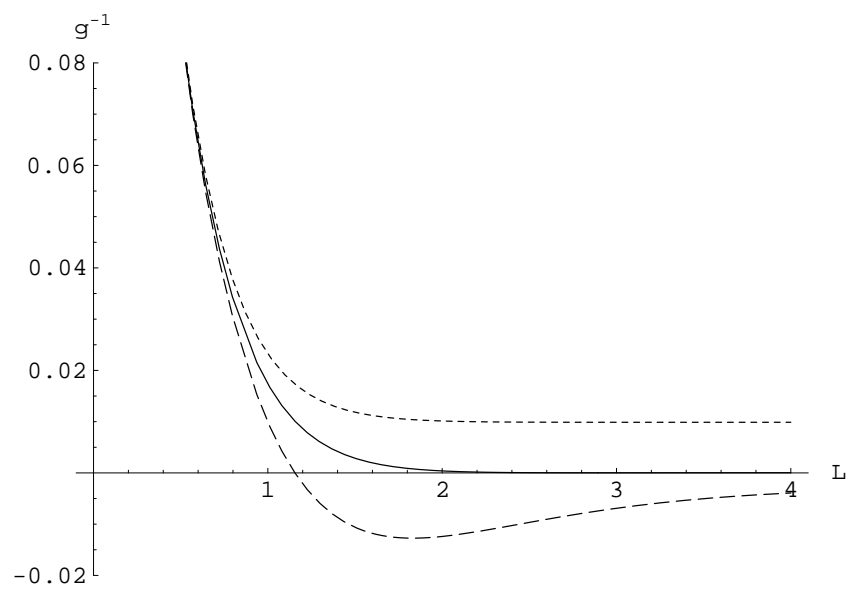

Figure 4: Inverse of the effective coupling constant $g_{22}^{-1}$, with $\lambda=30$ fixed, as a function of $L$ (in units of $m^{-1}$ ), for some values of $\beta$ (in units of $m^{-1}$ ): 2.4, 1.15 and 1.0 (dashed, full and dotted lines, respectively).

In Fig. 4, we plot $g_{22}^{-1}(L, \beta, \lambda)$ as a function of $L$, for some values of $\beta$ and a fixed value of $\lambda>\lambda_{c}^{(2)}$. We find that, in this example with $\lambda=30$, the minimum value of $g_{22}^{-1}$ vanishes for $\beta=$ $\beta_{d}^{(2)} \simeq 1.15 \mathrm{~m}^{-1}$ and is positive for $\beta>\beta_{d}^{(2)}$, no divergence of $g_{22}$ existing at temperatures above $\left(\beta_{d}^{(2)}\right)^{-1}$. Thus, the deconfining temperature is given by $T_{d}^{(2)}=\left(\beta_{d}^{(2)}\right)^{-1} \simeq 0.87 m$, for $\lambda=30$. The dependence of the deconfining temperature on $\lambda$ is presented in Fig. 5 .

4.2 $D=3$

We now investigate the effect of the temperature in the compactified 3- $D$ Gross-Neveu model by considering the coordinate $x_{3}$ (the imaginary time) compactified in a length $\beta=1 / T$. Taking, 


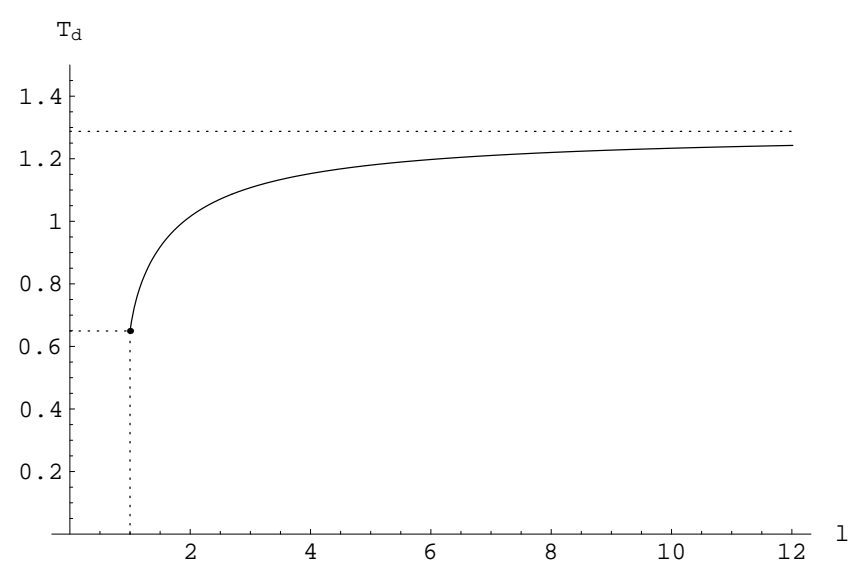

Figure 5: Deconfining temperature $T_{d}^{(2)}(\lambda)$ (in units of $m$ ), as a function of $l=\lambda / \lambda_{c}^{(2)}$; the horizontal dashed lines correspond to the limiting values $T_{\min }^{(2)} \simeq 0.65 \mathrm{~m}$ and $T_{\max }^{(2)} \simeq 1.29 \mathrm{~m}$.

again, $b_{1}=b_{2}=L^{-2}$ and fixing $b_{3}=\beta^{-2}$ in Eqs. (2.13 2.15), the $L-\beta$ dependent bubble diagram, is given by

$$
\begin{aligned}
\frac{\sum_{33}^{R}(L, \beta)}{m}= & \frac{1}{2 \pi}\left[\frac{2}{L} \log \left(1+e^{-L}\right)+\frac{1}{\beta} \log \left(1+e^{-\beta}\right)\right] \\
& -\frac{1}{2 \pi}\left[\frac{2}{1+e^{L}}+\frac{1}{1+e^{\beta}}\right] \\
& +\frac{1}{\pi}\left[G_{2}(L, L)+2 G_{2}(L, \beta)-4 G_{2}(L, 2 L)-4 G_{2}(L, 2 \beta)\right. \\
& \left.-4 G_{2}(2 L, \beta)+4 G_{2}(2 L, 2 L)+8 G_{2}(2 L, 2 \beta)\right] \\
& -\frac{1}{\pi}\left[2 G_{3}(L, L, \beta)-4 G_{3}(L, L, 2 \beta)-8 G_{3}(2 L, L, \beta)\right. \\
& \left.+8 G_{3}(2 L, 2 L, \beta)+16 G_{3}(2 L, L, 2 \beta)-16 G_{3}(2 L, 2 L, 2 \beta)\right],
\end{aligned}
$$

where $G_{2}(x, y)$ is given by Eq. (3.7) and the function $G_{3}(x, y, z)$ is defined by

$$
G_{3}(x, y, z)=\sum_{n, l, r=1}^{\infty} \exp \left(-\sqrt{x^{2} n^{2}+y^{2} l^{2}+z^{2} r^{2}}\right)\left[1-\frac{1}{\sqrt{x^{2} n^{2}+y^{2} l^{2}+z^{2} r^{2}}}\right]
$$

Note that, making $\beta \rightarrow \infty$, Eq. (4.3) reduces to $\Sigma_{32}^{R}(L)$, obtained from (3.6) with $L_{1}=L_{2}=L$.

As before, the increase of the temperature destroys the spatial confinement that exists for $\lambda \geq$ $\lambda_{c}^{(3)}$ at $T=0$. We can determine the deconfining temperature by searching for the value of $\beta(\lambda)$ for which the minimum of the inverse of the effective renormalized coupling constant, $g_{33}^{-1}(L, \beta, \lambda)=$ $\left(1+\lambda \Sigma_{33}^{R}(L, \beta)\right) / \lambda$, vanishes. For example, taking the specific case of $\lambda=110 \mathrm{~m}^{-1}$, we find $\beta_{d}^{(3)} \simeq 1.65 \mathrm{~m}^{-1}$ which corresponds to the deconfining temperature $T_{d}^{(3)} \simeq 0.61 \mathrm{~m}$; this result can be illustrated in a figure with the same pattern as that appearing in Fig. 4 for the $D=2$ case. The plot of $T_{d}^{(3)}(\lambda)$, as a function of $l=\lambda / \lambda_{c}^{(3)}$, has the same aspect as that in Fig. 5 with the limiting values $T_{d \min }^{(3)} \simeq 0.54 m$ and $T_{d \max }^{(3)} \simeq 0.87 m$. 
4.3 $D=4$

To look at the effect of finite temperature and determine the deconfining temperature for the fully compactified model in $D=4$, we need to compactify the imaginary time besides the spatial coordinates. With $b_{4}=\beta^{-2}$, measuring the lengths in units of $m^{-1}$, we find from Eqs. (2.12 2.15) that

$$
\begin{aligned}
\Sigma_{44}^{R}(L, \beta)= & m^{2}\left[6 H_{1}(2 L)-3 H_{1}(L)+2 H_{1}(2 \beta)-H_{1}(\beta)+6 H_{2}(L, L)+6 H_{2}(L, \beta)\right. \\
& -24 H_{2}(L, 2 L)-12 H_{2}(L, 2 \beta)-12 H_{2}(2 L, \beta)+24 H_{2}(2 L, 2 L)+24 H_{2}(2 L, 2 \beta) \\
& -4 H_{3}(L, L, L)-12 H_{3}(L, L, \beta)+24 H_{3}(L, L, 2 L)+48 H_{3}(L, 2 L, \beta) \\
& +24 H_{3}(L, L, 2 \beta)-48 H_{3}(L, 2 L, 2 L)-48 H_{3}(2 L, 2 L, \beta)-96 H_{3}(L, 2 L, 2 \beta) \\
& +32 H_{3}(2 L, 2 L, 2 L)+96 H_{3}(2 L, 2 L, 2 \beta)+8 H_{4}(L, L, L, \beta) \\
& -48 H_{4}(L, L, 2 L, \beta)-16 H_{4}(L, L, L, 2 \beta)+192 H_{4}(L, 2 L, 2 L, \beta) \\
& +96 H_{4}(L, L, 2 L, 2 \beta)-64 H_{4}(2 L, 2 L, 2 L, \beta) \\
& \left.-192 H_{4}(L, 2 L, 2 L, 2 \beta)+128 H_{4}(2 L, 2 L, 2 L, 2 \beta)\right] .
\end{aligned}
$$

where the functions $H_{1}, H_{2}$ and $H_{3}$ are given by Eqs. (3.9.3.11), and $H_{4}(x, y, z, w)$ is defined by

$$
\begin{aligned}
H_{4}(x, y, z, w)= & \frac{1}{2 \pi^{2}} \sum_{n, l, r, s=1}^{\infty}\left[K_{0}\left(\sqrt{x^{2} n^{2}+y^{2} l^{2}+z^{2} r^{2}+w^{2} s^{2}}\right)\right. \\
& \left.-\frac{K_{1}\left(\sqrt{x^{2} n^{2}+y^{2} l^{2}+z^{2} r^{2}+w^{2} s^{2}}\right)}{\sqrt{x^{2} n^{2}+y^{2} l^{2}+z^{2} r^{2}+w^{2} s^{2}}}\right] .
\end{aligned}
$$

As before, we can determine the deconfining temperature by searching for the value of $\beta(\lambda)$ for which the minimum of the inverse of the effective renormalized coupling constant, $g_{44}^{-1}(L, \beta, \lambda)=$ $\left(1+\lambda \Sigma_{44}^{R}(L, \beta)\right) / \lambda$, vanishes. For example, taking the specific case of $\lambda=620 \mathrm{~m}^{-2}$, we find $\beta_{d}^{(4)} \simeq 1.707 \mathrm{~m}^{-1}$ which corresponds to the deconfining temperature $T_{d}^{(3)} \simeq 0.59 \mathrm{~m}$. However, in the case of $D=4$, the lowest value of $T_{d \min }^{(4)}=0$; this means that, for $\lambda=\lambda_{c}^{(4)}$ the system is confined at $T=0$, but it becomes unconfined at any finite $T$; the upper bound of the deconfining temperature is $T_{d \max }^{(4)} \simeq 0.7 \mathrm{~m}$.

\section{Concluding remarks}

We have analyzed the $N$-component D-dimensional massive Gross-Neveu model with compactified spatial dimensions, both at zero and finite temperatures. The large- $N$ effective coupling constant $g$, for $T=0$, shows a kind of asymptotic freedom behavior, vanishing when the comapctification length tends to zero, irrespective to the value of the fixed coupling constant $\lambda$. In the strong coupling regime, where the fixed coupling constant is greater than some critical value, starting from small compactification lengths and increasing the size of the system, a divergence of the renormalized effective coupling constant appears at a given length, $L_{c}(\lambda)$, signalizing that the system gets spatial confined. When the temperature is raised, a deconfining transition occurs at a temperature $T_{d}(\lambda)$, as the minimum of the inverse of the renormalized effective coupling constant 
reaches zero. These general aspects of the model hold for arbitrary values of $D$, as explicitly shown for $D=2,3,4$.

It should be emphasized that these results are intrinsic of the model, do not emerging from any adjustment. The limit values of $L_{c}(\lambda)$ and $T_{d}(\lambda)$ depend only on the fermion mass. Thus, to get an estimate of these values we have to fix the parameter $m$. To do so, we consider the GrossNeveu model as an effective theory for the strong interaction (in which the gluon propagators have been shrank, similarly to the Fermi treatment of the week force) and take $m$ to be the constituent quark mass, $m \approx 350 \mathrm{MeV} \simeq 1.75 \mathrm{fm}^{-1}$ [15]. With such a choice, for the model with $D=3$ and both spatial coordinates compactified, we find $0.74 \mathrm{fm}<L_{c}(\lambda)<1.20 \mathrm{fm}$ and, correspondingly, $305 \mathrm{MeV}>T_{d}(\lambda)>189 \mathrm{MeV}$. These values should be compared with the experimentally measured proton charge diameter $(\approx 1.74 \mathrm{fm})[16]$ and the estimated deconfining temperature $(\approx 200 \mathrm{MeV})$ for hadronic matter [17]. A detailed analysis of such a comparison, for arbitrary dimension and in particular for $D=4$, will be presented elsewhere.

\section{Appendix: Analytical continuation of the multivariable zeta function}

Here, we summarize the steps to obtain Eq. (2.10). First, rewrite Eq. (2.9) in terms of sums over positive integers,

$$
\begin{aligned}
Z_{d}^{h^{2}}\left(\eta ; a_{1}, \ldots, a_{d}\right)= & \frac{1}{h^{2 \eta}}+2 \sum_{i=1}^{d} \sum_{n_{i}=1}^{\infty}\left(a_{i} n_{i}^{2}+h^{2}\right)^{-\eta} \\
& +2^{2} \sum_{i<j=1}^{d} \sum_{n_{i}, n_{j}=1}^{\infty}\left(a_{i} n_{i}^{2}+a_{j} n_{j}^{2}+h^{2}\right)^{-\eta}+\cdots \\
& +2^{d} \sum_{n_{1}, \ldots, n_{d}=1}^{\infty}\left(a_{1} n_{1}^{2}+\cdots+a_{d} n_{d}^{2}+h^{2}\right)^{-\eta}
\end{aligned}
$$

Using the identity,

$$
\frac{1}{\Delta^{\eta}}=\frac{1}{\Gamma(\eta)} \int_{0}^{\infty} d t t^{\eta-1} e^{-\Delta t}
$$

we get,

$$
\begin{aligned}
Z_{d}^{h^{2}}\left(\eta ; a_{1}, \ldots, a_{d}\right)= & \frac{1}{\Gamma(\eta)} \int_{0}^{\infty} d t t^{\eta-1} e^{-h^{2} t}\left[1+2 \sum_{i=1}^{d} T_{1}\left(t, a_{i}\right)+\right. \\
& \left.+2^{2} \sum_{i, j=1}^{d} T_{2}\left(t, a_{i}, a_{j}\right)+\cdots+2^{d} T_{d}\left(t, a_{1}, \ldots, a_{d}\right)\right]
\end{aligned}
$$

where,

$$
\begin{aligned}
T_{1}\left(t, a_{i}\right) & =\sum_{n_{i}=1}^{\infty} e^{-a_{i} n_{i}^{2} t} \\
T_{j}\left(t, a_{1}, \ldots, a_{j}\right) & =T_{j-1}\left(t, a_{1}, \ldots, a_{j-1}\right) T_{1}\left(t, a_{j}\right) \quad, j=2, \ldots, d .
\end{aligned}
$$

Considering the property of functions $T_{1}$,

$$
T_{1}\left(t, a_{i}\right)=-\frac{1}{2}+\sqrt{\frac{\pi}{a_{i} t}}\left[\frac{1}{2}+S\left(\frac{\pi^{2}}{a_{i} t}\right)\right]
$$


where

$$
S(x)=\sum_{n=1}^{\infty} e^{-n^{2} x}
$$

we find,

$$
\begin{aligned}
Z_{d}^{h^{2}}\left(\eta ; a_{1}, \ldots, a_{d}\right)= & \frac{\pi^{\frac{d}{2}}}{\sqrt{a_{1} \cdots a_{d}}} \frac{1}{\Gamma(\eta)} \int_{0}^{\infty} d t t^{\left(\eta-\frac{d}{2}\right)-1} e^{-h^{2} t}\left[1+2 \sum_{i=1}^{d} S\left(\frac{\pi^{2}}{a_{i} t}\right)\right. \\
& \left.+2^{2} \sum_{i<j=1}^{d} S\left(\frac{\pi^{2}}{a_{i} t}\right) S\left(\frac{\pi^{2}}{a_{j} t}\right)+\cdots+2^{d} \prod_{i=1}^{d} S\left(\frac{\pi^{2}}{a_{i} t}\right)\right] .
\end{aligned}
$$

Now, inserting in this expression the explicit form of the function $S(x)$ and using the following representation for Bessel functions of the third kind, $K_{v}$,

$$
2(a / b)^{\frac{v}{2}} K_{v}(2 \sqrt{a b})=\int_{0}^{\infty} d x x^{\nu-1} e^{-(a / x)-b x},
$$

we obtain Eq. (2.10).

\section{References}

[1] D.J. Gross and A. Neveu, Phys. Rev. D 10, 3235 (1974).

[2] C. de Calan, P.A. Faria da Veiga, J. Magnen and R. Séneor, Phys. Rev. Lett. 66, 3233 (1991).

[3] A. P. C. Malbouisson, J. M. C. Malbouisson, A. E. Santana and J. C. Silva, Phys. Lett. B 583, 373 (2004).

[4] F. C. Khanna, A. P. C. Malbouisson, J. M. C. Malbouisson, H. Queiroz, T. M. Rocha-Filho, A. E. Santana and J. C. Silva, Phys. Lett. B 624, 316 (2005).

[5] A. Chodos, R. L. Jaffe, K. Johnson, C. B. Thorn and V. F. Weisskopf, Phys. Rev D 9, 3471 (1974).

[6] C. A. Lutken and F. Ravndal, J. Phys. A: Math. Gen. 21, L793 (1988).

[7] A. P. C. Malbouisson and J. M. C. Malbouisson, J. Phys. A: Math. Gen. 35, 2263 (2002).

[8] A. P. C. Malbouisson, J. M. C. Malbouisson and A. E. Santana, Nucl. Phys. B 631, 83 (2002).

[9] L. M. Abreu, C. de Calan, A. P. C. Malbouisson, J. M. C. Malbouisson and A. E. Santana, J. Math. Phys. 46, 012304 (2005).

[10] J. C. da Silva, F. C. Khanna, A. Matos Neto and A. E. Santana, Phys. Rev. A 66, 052101 (2002).

[11] H. Queiroz, J. C. da Silva, F. C. Khanna, J. M. C. Malbouisson, M. Revzen and A. E. Santana, Ann. Phys. 317, 220 (2005); erratum and addendum, Ann. Phys. 321, 1274 (2006).

[12] E. Elizalde and A. Romeo, J. Math. Phys. 30, 1133 (1989).

[13] K. Kirsten, J. Math. Phys. 35, 459 (1994).

[14] E. Elizalde, S. D. Odintsov, A. Romeo, A. A. Bytsenko and S. Zerbini, Zeta regularization techniques with applications (World Scientific, Singapore, 1994); A. A. Bytsenko, G. Cognola, L. Vanzo and S. Zerbini, Phys. Rept. 266, 1 (1996); A. A. Bytsenko, G. Cognola, E. Elizalde, V. Moretti and S. Zerbini, Analytic aspects of quantum fields (World Scientific, River Edge, 2003).

[15] Particle Data Group, Phys. Lett. B 592, 1 (2004); see page 475.

[16] S. G. Karshenboim, Can. J. Phys. 77, 241 (1999).

[17] A. Smilga, Lectures on QCD, World Scientific, Singapore (2001), p. 279. 